\title{
Fatal pneumococcal septicaemia in coeliac disease
}

\author{
Donal J. O’Donoghue
}

Department of Medicine, University Hospital, Nottingham NG7 2UH, UK.

\begin{abstract}
Summary: A case of overwhelming pneumococcal septicaemia in a 34 year old man with coeliac disease and hyposplenism is reported. This previously unrecorded complication was fatal despite prompt treatment.
\end{abstract}

\section{Introduction}

Fulminant bacterial sepsis is a well recognized complication of splenectomy (Ferguson, 1982) even in the presence of extensive splenosis (Wilson \& Johnson, 1980). The overall frequency of serious bacterial infection in asplenic patients depends on their age, time since splenectomy and the underlying disease (Wilson \& Johnson, 1980). Children are at greater risk than adults, particularly in the first two years after splenectomy. In general, patients who have undergone splenectomy because of trauma have the lowest frequency $(1.5 \%)$ and those with thalassaemia $(25 \%)$ or lymphoreticular malignancy $(9.5 \%)$ the highest (Wilson \& Johnson, 1980). The mortality varies from over 50 to $70 \%$ of those infected.

Recently the hazards of serious infection in patients with inflammatory bowel disease and splenic atrophy have been recorded (Palmer et al., 1981). The present report describes overwhelming pneumococcal septicaemia in a patient with coeliac disease and splenic atrophy.

\section{Case report}

A 34 year old man with coeliac disease since childhood presented shocked and pyrexial. He did not adhere closely to his gluten free diet but was well until 2 days before admission. He described the gradual onset of a 'flu-like' illness with chills, rigors, generalized myalgia and mild headache.

His temperature was $41^{\circ} \mathrm{C}$, the pulse $120 / \mathrm{min}$ in sinus rhythm and his systolic pressure $80 \mathrm{~mm} \mathrm{Hg}$ by palpation. He had numerous bruises over his body and bled profusely from venepuncture sites. The neck was supple and there was no neurological deficit.

Correspondence and present address: D.J. O'Donoghue, B.Sc., M.B., M.R.C.P. Renal Unit, Manchester Royal Infirmary, Oxford Road, Manchester M13 9WL, UK. Accepted: 17 July 1985
The haemoglobin was $12.4 \mathrm{~g} / \mathrm{dl}$, white cell count $28 \times 10^{9} / 1$ and platelet count $14 \times 10^{9} / 1$. The plasma urea and electrolytes were normal. The prothrombin time was greater than 50 seconds and the partial thromboplastin time in excess of 100 seconds. A chest radiograph and electrocardiogram were normal apart from the sinus tachycardia. Blood cultures taken on admission later grew Streptococcus pneumoniae.

Intravenous fluids, hydrocortisone $(200 \mathrm{mg})$, cefuroxime $(750 \mathrm{mg})$ and gentamicin $(120 \mathrm{mg})$ were given immediately. Subsequently two units of fresh frozen plasma and 4 units of platelets were administered. Despite circulatory support with dopamine $(20 \mu \mathrm{g} / \mathrm{kg} /$ minute $)$ and dobutamine $(10 \mu \mathrm{g} / \mathrm{kg} /$ minute $)$ the blood pressure fell progressively and he died following a cardiac arrest 3 hours after admission.

Post-mortem examination revealed profuse petechiae and ecchymoses. The lungs were oedematous. The spleen was atrophic and weighed $25 \mathrm{~g}$ (normal $150-200 \mathrm{~g}$ ). There was sub-total villous atrophy of the mucosa of the small bowel. The adrenal glands were congested but not haemorrhagic. Post-mortem cultures from blood, lung and cerebrospinal fluid were negative. A primary site of infection was not found.

\section{Discussion}

This case illustrates the fulminant clinical course of overwhelming sepsis in patients with impaired splenic function despite prompt, medical intervention with antibiotics, fluids and inotropic support.

The causative organisms are usually encapsulated virulent pneumococci, meningococci or Haemophilus influenzae; but many other miscellaneous bacteria have been implicated in isolated reports. The onset can be explosive but is usually insidious beginning with a 'flu-like' illness and progressing over several days to frank septicaemia with circulatory collapse (Wilson \& Johnson, 1980). Although meningitis is common, a 
localized focus of infection is frequently absent even, as in this case, after a post-mortem examination. Severe disseminated intravascular coagulation is often a prominent feature and adrenal haemorrhage leading to the Waterhouse-Frederichsen syndrome well recognized. This intravascular coagulation is thought to be secondary to pneumococcal polysaccharide antigenantibody complexes aggregating platelets and activating complement (Wilson \& Johnson, 1980).

The prevalence of hyposplenism in coeliac disease is disputed, ranging from 16\% (McCarthy et al., 1966) to $77 \%$ (O'Grady et al., 1984). Its aetiology is unknown but the present case illustrates that these patients are at risk of overwhelming sepsis. In a recent study of 177 patients with coeliac disease impaired splenic function was present in $76.2 \%$ of untreated adults but improved after withdrawal of gluten and fell to $8.6 \%$ of patients with a normal small bowel mucosa (O'Grady

\section{References}

FERGUSON, A. (1982). Hazards of hyposplenism. British Medical Journal, 285, 1375.

MCCARTHY, C.F., FRASER, I.D., EVANS, K.T. \& READ, A.E. (1966). Lymphorectucular dysfunction in idiopathic streatorrhoea. Gut, 5, 140.

O'GRADY, J.G., STEVENS, F.M., HARDING, B., O'GORMAN, T.A., MCNICHOLL, B. \& McCARTHY, C. (1984). Hyposplenism and gluten-sensitive enteropathy. Gastroenterology, 87, 1326. et al., 1984). Interestingly, impaired splenic function remained common in those with persistent moderate or severe mucosal damage suggesting that complete regeneration of the mucosa must occur before hyposplenism is reversed.

The rapid tempo of this case underlines the importance of prophylaxis and early antibiotics in any susceptible patient. In spite of the blunted antibody response to pneumococcal vaccine in hyposplenism, it should be given since splenectomy increases the amount of antibody required to clear pneumococci from the blood stream (Ferguson, 1982). However, the optimum immunisation regime in hyposplenism is unknown. Therefore, any asplenic or hyposplenic patient with even a moderate fever or other signs of infection should be regarded as a potential case of overwhelming sepsis - blood cultures must be taken and speculative antibiotic treatment begun early.

PALMER, K.R., SHERRIFF, S.B., HOLDSWORTH, C.D. \& RYAN, F.P. (1981). Further experience of hyposplenism in inflammatory bowel disease. Quarterly Journal of Medicine, New Series L, 200, 463.

WILSON, S.A. \& JOHNSON, W.D. (1980). Infections complicating surgical or function splenectomy and gastroectomy. In Infections in the Abnormal Host, Grieco, M.H. (ed). p. 848. Yorke: New York. 